\title{
COMMUNITY ENGAGEMENT IN THE TELEHEALTH SERVICE FOR AGED PEOPLE WITH DIABETES: COVID-19 RESPONSE IN BANGLADESH
} \author{
Chowdhury5, Kazi Taznahel Sultana Ahona 6 \\ 1. University of South Asia, Dhaka, Bangladesh \\ 2. Abdul Malek Ukil Medical College, Bangladesh \\ 3. Chittagong Medical College, Bangladesh \\ 4. Crown Institute of Higher Education. Australia \\ 5. Centre for Injury Prevention and Research Bangladesh \\ 6. Jahangirnagar University, Savar, Dhaka, Bangladesh
}

Mohammad Shamsal Islam*1, Bimal Chandra Das², Mohammad Musa Meah3, Sanjoy Kumer Paul2, Ashim Kumar Saha², Reaz Uddin Chowdhury2, Mahfuz Ashraf4, Salim Mahmud

Correspondence: msislam009@gmail.com

\begin{abstract}
PURPOSE:

The purpose of this study is to present a better understanding of the specialized telehealth service in Bangladesh from the service provider and service recipients by aged people

\section{METHOD:}

Both quantitative and qualitative methods were used to collect data from Diabetes Mellitus (DM) patients. Data were collected by online telephone interviewing with an interview schedule. A total of 100 aged people with diabetes were selected purposively for a quantitative interview and 10 In-depth Interviews (IDIs) \& Key Informant Interviews (KIls) were conducted.
\end{abstract}

\section{RESULT:}

The majority of patients aged was between 61 to 68 years with a mean age of $63.6 \pm 7.01$ years. The difference of age of DM patients by sex was found statistically significant $(x 2=39.49, \mathrm{df}=31$; Cramer's $\mathrm{V}=.032 ; \mathrm{P}=<.003)$. The main source of information about digital health was: relatives $(55 \%)$, neighbors $(31 \%)$, television $(12 \%)$, newspaper $(10 \%)$, social media $(9 \%)$, and healthcare providers (6\%). Strong relationship was found between age of respondents and sources of information ( $\times 2=77.08$; Cramer's $V=.032$, df $=13$; Sig; $P=<.009$ ). About $59 \%$ of DM patients were benefited from telehealth services during COVID-19, however; they encountered some difficulties like effective access to digital technology, cost, and diagnosis facilities. About $83 \%$ of respondents suggest formalizing community engagement programs to extend the digital health services during a health emergency. The common barriers to the engagement of community people in digital health care are lack of social awareness, lack of peer group support, and gender disparities. Poor counseling, language barrier, bad internet signal, and lack of family members' support were the key barriers during teleconsultation services. 


\section{CONCLUSION:}

Telehealth has the potential to address critical health issues of aged people and effective community engagement may be the best option to reach older people with diabetes in Bangladesh during any health emergency.

\section{KEYWORDS}

Aged care, digital health, community engagement, Bangladesh

\section{INTRODUCTION}

The rising burden of chronic disease and the fasterincreasing rate of older people is to be considered the greatest challenge to the achievement of quality healthcare services. The increasing trend of older people is to be considered as a game-changer in the healthcare sectors and a key driver to change the demographic features of a country. It is projected that elderly people's growth in Bangladesh to be much faster (219\%) compared to some European countries such as Sweden (33\%), the UK (45\%), or Germany (66\%) in the period of 1990-2025. [1] eHealth is now considered an important tool to meet these challenges in developing countries like Bangladesh by engaging the community. For instance, technology in health care, welfare technology, mobile health care, and innovation of health care solutions. [2, 3, 4, 5] Community engagement and digital health have created an opportunity for all the stakeholders as part of the learning process by sharing responsibility and partnership for a transformative and holistic development approach. Community-based health development programs and social mobilization activities such as risk and protective factors, comprehensive and multi-level programs, effective and evidence-based interventions in the community setting are needed to consider designing the community development approach.

In recent times, the Government of Bangladesh (GoB) achieved remarkable progress in the transformation of digital health into COVID-19 response. As a part of digital health services to promote aged people health using telehealth services, the government provides a specialized telehealth service through the Diabetic Association of Bangladesh (BADAS) for aged people with diabetes. It is being evident that diabetes is a significant contributor to deaths and complications among people infected by the nCov-19 in Bangladesh.
Government of Bangladesh has introduced digital health strategy as well as tele-health care service provision for Bangladeshi people in 2019 in pursuing resolution 71.7 of the world health assembly. . Recently government of Bangladesh has introduced tele-healthcare services in the 2 specialized hospitals (Bangabandhu Sheikh Mujib Medical University and National Institute of Cardiovascular Diseases), 3 district hospitals (Shatkhira, Nilphamari and Gopalganj) and 3 sub-district hospitals (Pirgonj, Dakope and Debhata). Around 15 digital healthcare service providers are working in the country wide. This learning process can be useful as an effective tool for determining the future course of action and steps will have intended to support elders in the community to use digital health technologies as easy access and such a process usually strengthens the skills and capacity of the community members. Simultaneously, this process helps both parties (stakeholders and actors) to build confidence through cooperative learning and confidence-building to improve the conditions of target groups and level of knowledge. [ 7 , 8] In this context, we need a comprehensive study of these stakeholders and how they act about each other is warranted, particularly in the context of digital health care services for aged people in Bangladesh. With the recognition that information and communications technologies aim to attain new opportunities and challenges for the achievement of Sustainable Development Goals (SDGs), there is a growing consensus in the global health community that the strategic and innovative use of digital and cutting-edge information and communications technologies will be an essential enabling factor towards other healthcare initiatives such as universal health coverage, health emergencies, and health wellbeing [9]. Digital health become an essential part of daily health care services and government is planning to amendment the healthcare services and transform it into digital care especially for aged people within on-going healthcare facilities. BADAS ongoing tele-healthcare 
services is to consider model initiative to address the diabetes care for aged people in Bangladesh.

The purpose of this study is to present a better understanding of the specialized telehealth service in Bangladesh from the service provider and service recipients by aged people.

\section{METHODS}

Mixed methods were used to collect data from DM patients. Data were collected by online telephone interviewing with an interview schedule. A total of 100 aged people with diabetes were selected purposively for Quantitative interviews and 10 IDIs \& Klls were conducted.

\section{SETTING THE SCENARIO:}

The Heath system of Bangladesh is working with 600 public hospitals, 16,438 community clinics, and 30,000 satellite clinics to provide healthcare services to around 165 million people. These statistics show that 0.47 doctors per 1,000 populations. [10] Diabetic Association of Bangladesh (BADAS) is a non-profit organization is delivered diabetes care services around half of the people with diabetes in Bangladesh. The organization manages over 100 institutions, covering small to large hospitals and rendering services to primary, secondary, and tertiary care across all disciplines throughout the country. The research was conducted at 4 BADAS hospitals at the district level and data were collected from a patient who attended services and treatment from those hospitals. Diabetic The aged people who attended BADAS hospital for diabetic treatment were interviewed as a key informant for this research. Furthermore, $10 \mathrm{IDIs}$ were also conducted for aged people with DM.

\section{STUDY POPULATION, SAMPLE SIZE AND SAMPLING:}

Both quantitative and qualitative data were collected through a sample survey and in-depth interviews, respectively. In sample survey those variables were included which had primarily provided quantitative information, while qualitative data were express feelings, perceptions, and opinions. The patient aged above 65 years were purposively selected to collect information for this study.

The interview schedule was pretested and obtained data were analyzed for determining reliability. Face validity of the questions was determined by research an expert.
Sample size was finalized using a standard formula (Fisher, et al., 1991), which was around 100.

\section{DESIGN AND DEVELOP OF RESEARCH TOOLS:}

Both structured and open-ended questions were used in quantitative survey while for in-depth interview data were collected with the help of an interview guide, which included unstructured questions having option to continue to instantly formulate questions for collecting meaningful data. Each in-depth interview has transcribed within 24 hours of interview lest information is lost. In-depth interview respondent's diabetes patients, service providers, and policy makers.

\section{DATA COLLECTION, QUALITY CONTROL, AND DATA MANAGEMENT:}

Data (open and close-ended questions) were collected over 1 month (November 1 to November 30, 2020). A total of 100 patients were interviewed using an interview guide. A workshop was conducted with the research assistants to better understanding the study objectives and data collection procedures. The study format and interview schedule were given to diabetologists and public health experts for determining content validity. Their comments were incorporated for updating the research instruments. The filled-in formats and interview schedules were edited immediately after the collection.

\section{DATA ANALYSIS PLAN:}

SPSS statistical package was used for analyzing quantitative data. Survey data was coded and entered into the computer for analysis. Univariate and bivariate tables were used for analysis purposes. Data analysis included frequency distribution, cross-tabulation, corelation, and association and, statistically significant tests between variables (X2, p-value, and $\mathrm{Cl}$ ). The qualitative data were analyzed manually using the content analysis technique. Categorization was done according to themes and sub-themes.

\section{ETHICAL CONSIDERATION}

Verbal consent was obtained from each participant after explaining the purpose and nature of the research. Participation in the study was voluntary and participants were informed of their right to quit/refuse their participation at any stage of the study if they do not want to participate. Moreover, the confidentiality of the information was assured by using an anonymous consent form. 


\section{SOCIO-DEMOGRAPHIC INFORMATION OF DM PATIENTS:}

The study revealed that a large number (73\%) of diabetes patients aged between 61 to 68 years with a mean age of $63.6 \pm 7.01$ years, ranging from 55 to 84 years old, which means a large number of diabetes patients were elderly and few were very aged. A total of 40 males and 60 females' diabetes patients were included in this study. The difference of age of DM patients by sex was found statistically significant at .003 level $\left(x^{2}=39.49\right.$, df $=31$; Cramer's $V=.032)$. The studied most of the DM patients were Muslim (91.8) whereas $8.2 \%$ were Hindu. The majority (75\%) of the DM patient family size was 4-5 and level of education was up to primary (33\%), High school $(30.60 \%)$, SSC (10\%), HSC (4.9\%), Graduate (2.75\%), Postgraduate $(.5 \%)$ and Illiterate $(20.5 \%)$ respectively. About $42.5 \%$ of the DM patients' occupation was housewife, $22.2 \%$ were service holder, $10.3 \%$ were businessman, $8.8 \%$ were retired person, $5.5 \%$ were old/aged and $4.3 \%$ were a farmer and $2.5 \%$ were day labor. About $88.5 \%$ DM patients were married, $7 \%$ were the widow and remaining $.5 \%$ was divorced. On average DM patients came hospital from a $42 \mathrm{KM}$ distance to receive treatment and services.

\section{TABLE 1: PERCENTAGE DISTRIBUTION OF AGE OF THE RESPONDENTS BY GENDER}

\begin{tabular}{|l|l|l|l|}
\hline \multirow{2}{*}{ AGE CATEGORIES } & MALE & FEMALE \\
& N=40 & N0 & TOTAL \\
& Percentage & Percentage & Percentage \\
\hline $55-60$ years & $8.5 \%$ & $30 \%$ & $20 \%$ \\
\hline 61-65 years & $40 \%$ & $47 \%$ & $44 \%$ \\
\hline $66-70$ years & $24 \%$ & $14 \%$ & $19 \%$ \\
\hline $71-80$ years & $20 \%$ & $6 \%$ & $13 \%$ \\
\hline $81-85$ years & $7.5 \%$ & $3 \%$ & $4 \%$ \\
\hline Total & 100.0 & 100.0 & 100.0 \\
\hline Mean & 63.01 & 63.96 & 63.52 \\
\hline Median & 66.90 & 67.70 & 67.80 \\
\hline St. deviation & 7.11 & 7.16 & 7.14 \\
\hline$x^{2}=39.49 ;$ Cramer's V= $=031, \mathrm{df}=9 ;$ Sig; $\mathrm{P}=<.003$ & \\
\hline
\end{tabular}

\section{COMMUNITY ENGAGEMENT AND AGED PEOPLE PERCEPTION OF DIGITAL HEALTH SERVICES:}

About $66 \%$ of the respondents were informed about telehealth services and about $58 \%$ of the elderly people have received some sort of telehealth services during COVID-19. About $55 \%$ of aged people heard about digital health from their relatives whereas $31 \%$ were informed about digital health care services from their neighbors. People also heard from mass media like television (12\%), newspaper (10\%), Facebook (9\%) whereas $6 \%$ were informed by formal healthcare providers. A strong relationship is found between the age of respondents and sources of information. The formal sources of information obtained by respondents progressively increase with the increase of their age $\left(x^{2}=77.08\right.$; Cramer's $V=.032$, df $=13$; Sig; $P=<.009$ ). Possible reasons could be that relatively older people chose to stay at home due to COVID-19 and were more likely to watch TV, follow radio programs and read the newspaper to get updates on the COVID-19 pandemic while passing time at home. The interesting feature is that about $78 \%$ of aged people welcome digital health services like telehealth services of BADAS and among them $59 \%$ had experienced receiving telehealth service during COVID and lockdown situations. The vast majority (83\%) suggested formalizing community engagement programs to extend the digital health services during a health emergency. About $27 \%$ of aged people had faced various difficulties in receiving treatment during lockdown using digital health platforms. The major difficulties were access to digital technology (42\%), diagnosis of diseases (36\%), and buying of prescribed medicine (22\%) due to financial crisis. BADAS established its digital care center in 2018 aimed to access health care for aged people. The big concern is that about $56 \%$ of users 
are not happy with their services and suggested more community engagement for the effective utilization of digital health services. Recommendations were sought from the respondents for the improvement of services of digital health. In response to this, some suggestions were put forward by the respondent. The highest percentage of respondents suggested making more facilitating digital care (56\%), followed by providing better diagnosis facilities (43\%), effective advocating for people with diabetes (38\%), promoting community awareness (32\%), and strong community engagement especially community leaders (29\%). About $36 \%$ of participants believe that the recent COVID-19 shows high demand for community engagement. Participants were informed about digital health care for aged people from different sources such as Physician (46\%); Television (21\%), and Neighbors (45\%). Our data show that a large proportion (56\%) of respondents were not happy with the existing quality of services. The aged people think that telehealth services support them to prevent any sort of direct physical contact (49\%), provide continuous care to the older community (46\%), and reduce morbidity and mortality in COVID-19 (44\%). The important issue is that a large number of respondents (58\%) are informed about government digital services. Common barriers to engagement of community people in digital health care for aged people such as lack of social awareness (55\%), lack of peer group support (44\%), and gender disparities (32\%) are notable. In general, a health educator or counselor provides counseling on DM complications, the importance of regular follow-up and visits of referring places, how to control sugar levels, how to maintain a healthy lifestyle, and advice for the next appointment. Respondents were informed about diabetes treatment from doctors (73\%), quacks (12\%), rural physicians (52\%), and allied health personnel (48\%). A digital platform like social media also plays a vital role to create strong community engagement and help to improve their health-seeking behavior at the individual and community level. Majority of the respondents opined that development of infrastructure, connectivity, quality of digital healthcare, and validation of digital health care maybe crucial steps to engage the community.

\section{FINDINGS FROM QUALITATIVE INTERVIEWS:}

In order to understand aged people expectation and service providers' views in response to digital healthcare we tried to comprehend common persons' and occupational groups' perception of tele-healthcare for aged diabetes patients. We conducted in depth interview. Some participants believe that most of the aged people have rejected digital healthcare because they prefer going to specialists or qualified physicians physically for treatment. From our KIls and IDIs data, we found that most of the respondent's mention barriers to receive digital healthcare. The majority of the participant's mention health education barriers like poor counseling, poor time allocation, language barriers (sometimes they couldn' $\dagger$ understand the medical terminology using by a physician or allied health personnel), and lack of support from family members during telehealth conversations. Participants also mention financial barriers that negatively affect telehealth services and treatment (internet or mobile charge). Some aged people said that they do not even have money for a daily living how could even be possible to continue such long time-costly treatment over a telephone call. The participants hold the view that for lack of financial constraints they are not able to buy smart phone and unable to get digital healthcare.

Aged DM patients mentioned that community people have a lack of awareness such as an ignorant attitude to telehealth services, and in some cases, patients have less attention to the health counseling. Patient guardian has very low interest on telehealth services because they think that 'there are many gossiping but no-talk about diabetes. Poor communication between patients and service providers is the key barrier to provide telehealth care. Respondent suggests to effective awareness program about telehealth services, meaningful community engagement, cheap internet service, easy apps facility, and improve language barriers to deliver the counseling to aged people.

Perceptions were also measured through informal discussion and few structured questions. Most of the people perceive that digital platform is not good for quality healthcare especially for aged diabetes people. Majority of participates still believe in herbal treatment for their diabetes but they opined that modern treatment of diabetes is needed and service facilities should be made available. They have suggested arranging a one-day training session for aged people at community level on how to use digital platform to get easy treatment in the older age.

\section{DISCUSSION}

The primary goal of the study was to understand the service recipients' opinions about digital health and the 
importance of community engagement service of BADAS for aged people during COVID-19. Health emergencies like COVID-19 push aged people to manage home quarantine and receive the tele-healthcare services provided by BADAS in a community setting. Government Digital Health Atlas (DHA) and BADAS digital health care is an initiative for aged people is parallel activities to reach aged people in Bangladesh. BADAS started its telehealth care for aged people in 2018 aimed at helping the aged people to reduce the suffering and make the services available. BADAS as a non-profit organization is trying to minimize the gap of its program and to make it user-friendly to aged people. Evidence shows that majority of the aged people is missing out on community engagement for digital health care initiative. Digital health care and community engagement is a new era for aged people of Bangladesh and COVID-19 is the best time to provide such services to people with diabetes. From our data, we found that the mean age of respondents was $63.6 \pm 7.01$ years and the majority of the respondents were female. The age of aged people with diabetes by sex was found statistically significant. Possible reasons could be that relatively older people chose to stay at home due to COVID-19 and were more likely to watch TV, follow radio programs and read the newspaper to get updates on the COVID-19 pandemic. Aged diabetes patients on average travel 42 kilometers to get physical services from the diabetes hospitals. The majority of the aged people like to welcome digital health care services and $59 \%$ of aged people was benefited from BADAS telehealth services during COVID-19 and lockdown. Our study finding was similar to Nazim et al., (2020) [10] and they found that $52 \%$ of aged people were benefited from telehealth services during COVID-19. Recent studies show that digital health that connects diabetes patients with healthcare providers was level 2 using the video mode. [11] This facility is absent in the context of Bangladeshi digital healthcare. The majority of the aged diabetes patient who is living in the rural remote areas do not have such smart phone facility to conduct consultation with physician y using the video mode. On the other hand, most of advance level (3B) users using the CGM devices which provide active monitoring diagnosis and treatment based on the timely clinical judgement. [12]

Aged people with diabetes had faced some difficulties during telehealth services such as access to digital technology, cost, and diagnosis facilities. Our study findings were familiar with the findings of Tan et al., (2020) [13] and they reveal that aged people face barriers to the adoption of telehealth services such as cost, access, trust of technology, privacy concern, and user interface during COVID-19. Community engagement approach and telehealthcare for aged people with diabetes are innovative healthcare services to remote underprivileged people in Bangladesh. Our data shows that community engagement is poor in Bangladesh and the vast majority (83\%) suggested formalizing community engagement programs to extend the digital health services during any health emergency. Community engagement is an effective tool to provide quality services to the service recipients and about $56 \%$ were not happy with present telehealth services. The use of CGM with synchronized data sharing makes diabetes educators and clinicians easy to make decisions and fix the patient's problem during the pandemic. This wearable equipment classified at level 3B because it could empower patients and families. The patients and family can do active monitoring, recording the glycemic status day by day, transmit the data to the healthcare, and do early specific diagnosis by reminder feature of hypoglycemia and hyperglycemia alarm. [14] The common barriers are to engage community people in digital health care for the aged people are poor social awareness, the low interest of peer group support, and gender disparities. Poor counseling, language barrier, bad internet signal, and lack of family members' support are key barriers to deliver teleconsultation services. Qualitative findings also show poor counseling, insufficient allocation of time, language barriers, and family members' ignorant attitudes are the key barriers to receive quality service from tele-healthcare. Furthermore, the financial barrier is the key issue for aged people with diabetes, and in some cases, participants have less interest to attend telehealth services due to the high cost of the internet. Respondents suggest promoting the quality counseling session, continuing effective community awareness programs, meaningful community engagement activities, and enable access to tele-healthcare are the key factors to improve the telehealthcare for aged in Bangladesh.

\section{CONCLUSION}

Aged people with diabetes are struggled to receive quality diabetes healthcare through a digital platform in Bangladesh. BADAS initiative opens a new window of opportunity for aged people to receive diabetes healthcare by staying at home. Telehealth services during any health emergency play a vital role to address the critical health issues of unprivileged aged people living in rural areas. Digital health strategy and proactive 
community engagement may support aged diabetes people health care access during self-confinement.

\section{ACKNOWLEDGMENT:}

We would like to acknowledge Professor Abul Hasnat Golum Quddus, Honorary Director (Dr. Ahmadur Rahman Research Centre) the University of Chittagong for his comments on the very initial version.

\section{References:}

1. Uddin, M. T., Islam, M. N. and Kabir, A., "Demographic Dependency of Aging Process in Bangladesh", Pakistan Academy of Science, 2012; Nol. 49 No. 3, pp. 209-218

2. Bygstad B, Hanseth $O$. Governing e-Health infrastructures: dealing with tensions. Proceedings of the 37th International Conference on Information Systems. Dublin: AIS eLibrary; 2016

3. Korhonen $\mathrm{O}$, Isomursu $\mathrm{M}$. Identifying personalization in a care pathway: a single-case study of a Finnish healthcare service provider. Proceedings of the 25th European Conference on Information Systems (ECIS). Guimarães: AIS eLibrary. 2017:828-841.

4. Meng F, Guo X, Peng Z, Zhang X, Vogel D. The routine use of mobile health services in the presence of health consciousness. Electron Commer R A. 2019; 35:100847.

5. Petrakaki D. Producing communal health through self care: the emergence of digital patient activism. Proceedings of the 25th European Conference on Information Systems (ECIS). Guimarães: AIS eLibrary; 2017.

6. Broadband Commission for Sustainable Development, Digital Health: A call for government leadership and cooperation between ICT and health, Broadband Commission for Sustainable Development, ITU and UNESCO, February 2017, accessed 17 August 2018

7. Barua, B.P (2009). Participatory research, NGOs, and grassroots development: challenges in rural Bangladesh. In D. Kapoor and S. Jordon (Eds.), Education, Participatory Action Research and Social Change: International Perspective. New York: Palgrave Macmillan.

8. Rahman, M.A. (1993). People's Self Development: Perspective on Participatory Action Research: A Journey through Experience. London; Zed Books/and Dhaka: The University Press.

9. World Health Organization (WHO), 2017
10. Mohiuddin AK. An extensive review of patient healthcare service satisfaction in Bangladesh. Adesh Univ J Med Sci Res 2020;2(1):5-16.

11. Purnamayanti, Ni K. D.; Wicaksana, Anggi L. 1,2, Digital Health Services among Patients with Diabetes during the COVID-19 Pandemic, Indian Journal of Endocrinology and Metabolism: March-April 2021 Volume 25 - Issue 2 - p 86-91 doi: 10.4103/ijem.ijem_153_21

12. Ciardullo S, Zerbini F, Perra S, Muraca E, Cannistraci R, Lauriola M, et al Impact of diabetes on COVID-19related in-hospital mortality:A retrospective study from Northern Italy J Endocrinol Invest. 2021;44:843-50.

13. Monaghesh, E., Hajizadeh, A. The role of telehealth during COVID-19 outbreak: a systematic review based on current evidence. BMC Public Health 20, 1193 (2020). https://doi.org/10.1 186/s12889-020-09301-4

14. United Nation. COVID-19 impact on treatment for chronic illness revealed. Available from: https://news.un.org/en/story/2020/09/1071732.P ublished 2020 